\title{
Division Algebras and Supersymmetry I
}

\author{
John C. Baez and John Huerta \\ Department of Mathematics \\ University of California \\ Riverside, CA 92521 USA
}

December 29, 2009

\begin{abstract}
Supersymmetry is deeply related to division algebras. For example, nonabelian Yang-Mills fields minimally coupled to massless spinors are supersymmetric if and only if the dimension of spacetime is 3, 4, 6 or 10 . The same is true for the Green-Schwarz superstring. In both cases, supersymmetry relies on the vanishing of a certain trilinear expression involving a spinor field. The reason for this, in turn, is the existence of normed division algebras in dimensions two less, namely 1, 2, 4 and 8: the real numbers, complex numbers, quaternions and octonions. Here we provide a self-contained account of how this works.
\end{abstract}

\section{Introduction}

There is a deep relation between supersymmetry and the four normed division algebras: the real numbers $\mathbb{R}$, the complex numbers $\mathbb{C}$, the quaternions $\mathbb{H}$, and the octonions $\mathbb{O}$. This is visible in the study of superstrings, supermembranes, and supergravity, but perhaps most simply in supersymmetric Yang-Mills theory. In any dimension, we may consider a Yang-Mills field coupled to a massless spinor transforming in the adjoint representation of the gauge group. These fields are described by this Lagrangian:

$$
L=-\frac{1}{4}\langle F, F\rangle+\frac{1}{2}\left\langle\psi, \not D_{A} \psi\right\rangle .
$$

Here $A$ is a connection on a bundle with semisimple gauge group $G, F$ is the curvature of $A, \psi$ is a $\mathfrak{g}$-valued spinor field, and $\not_{A}$ is the covariant Dirac operator associated with $A$. It is well-known that this theory is supersymmetric if and only if the dimension of spacetime is $3,4,6$, or 10 . Our goal here is to present a self-contained proof of the 'if' part of this result, based on the theory of normed division algebras.

This result goes back to the work of Brink, Schwarz, and Sherk 3 and others. The book by Green, Schwarz and Witten [10] contains a standard proof based on the properties of Clifford algebras in various dimensions. But Evans [7] has shown that the supersymmetry of $L$ in dimension $n+2$ implies the existence of a normed division algebra of dimension $n$. Conversely, Kugo and Townsend [12] showed how spinors in dimension 3, 4, 6, and 10 derive special properties from the normed division algebras $\mathbb{R}, \mathbb{C}, \mathbb{H}$ and $\mathbb{O}$. They formulated a supersymmetric model in 6 dimensions using the quaternions, $\mathbb{H}$. They also speculated about a similar formalism in 10 dimensions using the octonions, $\mathbb{O}$.

Shortly after Kugo and Townsend's work, Sudbery [17] used division algebras to construct vectors, spinors and Lorentz groups in Minkowski spacetimes of dimensions 3, 4, 6, and 10. He then refined his construction with Chung [4], and with Manogue [13] he used these ideas to give an octonionic proof 
of the supersymmetry of the above Lagrangian in dimension 10. This proof was later simplified by Manogue, Dray and Janesky [5]. In the meantime, Schray [14] applied the same tools to the superparticle.

All this work has made it quite clear that normed division algebras explain why the above theory is supersymmetric in dimensions $3,4,6$, and 10 . Technically, what we need to check for supersymmetry is that $\delta L$ is a total divergence with respect to the supersymmetry transformation

$$
\begin{aligned}
& \delta A=\epsilon \cdot \psi \\
& \delta \psi=\frac{1}{2} F \epsilon
\end{aligned}
$$

for any constant spinor field $\epsilon$. (We explain the notation here later; we assume no prior understanding of supersymmetry or normed division algebras.) A calculation that works in any dimension shows that

$$
\delta L=\operatorname{tri} \psi+\text { divergence }
$$

where tri $\psi$ is a certain expression depending in a trilinear way on $\psi$ and linearly on $\epsilon$.

So, the marvelous fact that needs to be understood is that tri $\psi=0$ in dimensions $3,4,6$, and 10 , thanks to special properties of the normed division algebras $\mathbb{R}, \mathbb{C}, \mathbb{H}$ and $\mathbb{O}$. Indeed, this fact is responsible for supersymmetry, not only for Yang-Mills fields in these dimensions, but also for superstrings! The same term tri $\psi$ shows up as the obstruction to supersymmetry in the GreenSchwarz Lagrangian for classical superstrings [9, 10]. So, the vanishing of this term deserves to be understood: clearly, simply, and in as many ways as possible.

Unfortunately, many important pieces of the story are scattered throughout the literature. The treatment of Deligne and Freed [6] is self-contained, and it uses normed division algebras, but it does not use 'purely equational reasoning': it proves tri $\psi=0$ by first showing that the double cover of the Lorentz group acts transitively on the set of nonzero spinors in dimensions $3,4,6$, and 10 . While this geometrical argument is beautiful and insightful, a purely equational approach has its own charm. The line of work carried out by Fairlie, Manogue, Sudbery, Dray, and collaborators [5, 8, 13, 14 has shown that the equation tri $\psi=0$ can be derived from the complete antisymmetry of another trilinear expression, the 'associator'

$$
[a, b, c]=(a b) c-a(b c)
$$

in the normed division algebra. Our desire here is to merely present this argument as clearly as we can.

So, here we present an equational proof that tri $\psi=0$ in dimensions $3,4,6$, and 10 , based on the complete antisymmetry of the associator for the normed division algebras $\mathbb{K}=\mathbb{R}, \mathbb{C}, \mathbb{H}$ and $\mathbb{O}$. In Section 2 we review the properties of normed division algebras that we will need. In Section 3 we start by recalling how to interpret vectors as $2 \times 2$ hermitian matrices with entries in $\mathbb{K}$, and spinors as elements of $\mathbb{K}^{2}$. We then use this language to describe the basic operations involving vectors, spinors and scalars. These include an operation that takes two spinors $\psi$ and $\phi$ and forms a vector $\psi \cdot \phi$, and an operation that takes a vector $A$ and a spinor $\psi$ and forms a spinor $A \psi$. In Section 4 we prove the fundamental identity that holds only in Minkowski spaces of dimensions 3, 4, 6 and 10:

$$
(\psi \cdot \psi) \psi=0
$$

Following Schray [14, we call this the ' $3-\psi$ 's rule'. In Section 5 we introduce a little superalgebra, and explain why we should treat $\mathbb{K}$ as an 'odd', or 'fermionic', super vector space. In Section 6 we formulate pure super-Yang-Mills theory in terms of normed division algebras, completely avoiding the use of gamma matrices. We explain how the term tri $\psi$ arises as the obstruction to supersymmetry in this theory. Finally, we use the $3-\psi$ 's rule to prove that tri $\psi=0$ in dimensions $3,4,6$ and 10 . 


\section{Normed Division Algebras}

By a classic theorem of Hurwitz [11, there are only four normed division algebras: the real numbers, $\mathbb{R}$, the complex numbers, $\mathbb{C}$, the quaternions, $\mathbb{H}$, and the octonions, $\mathbb{O}$. These algebras have dimension 1, 2, 4, and 8. For an overview of this subject, including a Clifford algebra proof of Hurwitz's theorem, see [1. Here we introduce the bare minimum of material needed to reach our goal.

A normed division algebra $\mathbb{K}$ is a (finite-dimensional, possibly nonassociative) real algebra equipped with a multiplicative unit 1 and a norm $|\cdot|$ satisfying:

$$
|a b|=|a||b|
$$

for all $a, b \in \mathbb{K}$. Note this implies that $\mathbb{K}$ has no zero divisors. We will freely identify $\mathbb{R} 1 \subseteq \mathbb{K}$ with $\mathbb{R}$.

In all cases, this norm can be defined using conjugation. Every normed division algebra has a conjugation operator - a linear operator $*: \mathbb{K} \rightarrow \mathbb{K}$ satisfying

$$
a^{* *}=a, \quad(a b)^{*}=b^{*} a^{*}
$$

for all $a, b \in \mathbb{K}$. Conjugation lets us decompose each element of $\mathbb{K}$ into real and imaginary parts, as follows:

$$
\operatorname{Re}(a)=\frac{a+a^{*}}{2}, \quad \operatorname{Im}(a)=\frac{a-a^{*}}{2} .
$$

Conjugating changes the sign of the imaginary part and leaves the real part fixed. We can write the norm as

$$
|a|=\sqrt{a a^{*}}=\sqrt{a^{*} a} .
$$

This norm can be polarized to give an inner product on $\mathbb{K}$ :

$$
(a, b)=\operatorname{Re}\left(a b^{*}\right)=\operatorname{Re}\left(a^{*} b\right) .
$$

The algebras $\mathbb{R}, \mathbb{C}$ and $\mathbb{H}$ are associative. The octonions $\mathbb{O}$ are not. Yet they come close: the subalgebra generated by any two octonions is associative. Another way to express this fact uses the associator:

$$
[a, b, c]=(a b) c-a(b c),
$$

a trilinear map $\mathbb{K} \otimes \mathbb{K} \otimes \mathbb{K} \rightarrow \mathbb{K}$. A theorem due to Artin [15] states that for any algebra, the subalgebra generated by any two elements is associative if and only if the associator is alternating (that is, completely antisymmetric in its three arguments). An algebra with this property is thus called alternative. The octonions $\mathbb{O}$ are alternative, and so of course are $\mathbb{R}, \mathbb{C}$ and $\mathbb{H}$ : for these three the associator simply vanishes!

In what follows, our calculations make heavy use of the fact that all four normed division algebras are alternative. Besides this, the properties we require are:

Proposition 1. The associator changes sign when one of its entries is conjugated.

Proof. Since the subalgebra generated by any two elements is associative, and real elements of $\mathbb{K}$ lie in every subalgebra, $[a, b, c]=0$ if any one of $a, b, c$ is real. It follows that $[a, b, c]=[\operatorname{Im}(a), \operatorname{Im}(b), \operatorname{Im}(c)]$, which yields the desired result.

Proposition 2. The associator is purely imaginary.

Proof. Since $(a b)^{*}=b^{*} a^{*}$, a calculation shows $[a, b, c]^{*}=-\left[c^{*}, b^{*}, a^{*}\right]$. By alternativity this equals $\left[a^{*}, b^{*}, c^{*}\right]$, which in turn equals $-[a, b, c]$ by the above proposition. So, $[a, b, c]$ is purely imaginary. 
For any square matrix $A$ with entries in $\mathbb{K}$, we define its trace $\operatorname{tr}(A)$ to be the sum of its diagonal entries. This trace lacks the usual cyclic property, because $\mathbb{K}$ is noncommutative, so in general $\operatorname{tr}(A B) \neq \operatorname{tr}(B A)$. Luckily, taking the real part restores this property:

Proposition 3. Let $a, b$, and $c$ be elements of $\mathbb{K}$. Then

$$
\operatorname{Re}((a b) c)=\operatorname{Re}(a(b c))
$$

and this quantity is invariant under cyclic permutations of $a, b$, and $c$.

Proof. Proposition 2 implies that $\operatorname{Re}((a b) c)=\operatorname{Re}(a(b c))$. For the cyclic property, it then suffices to prove $\operatorname{Re}(a b)=\operatorname{Re}(b a)$. Since $(a, b)=(b, a)$ and the inner product is defined by $(a, b)=\operatorname{Re}\left(a b^{*}\right)=$ $\operatorname{Re}\left(a^{*} b\right)$, we see:

$$
\operatorname{Re}\left(a b^{*}\right)=\operatorname{Re}\left(b^{*} a\right) .
$$

The desired result follows upon substituting $b^{*}$ for $b$.

Proposition 4. Let $A, B$, and $C$ be $k \times \ell, \ell \times m$ and $m \times k$ matrices with entries in $\mathbb{K}$. Then

$$
\operatorname{Re} \operatorname{tr}((A B) C)=\operatorname{Re} \operatorname{tr}(A(B C))
$$

and this quantity is invariant under cyclic permutations of $A, B$, and $C$. We call this quantity the real trace $\operatorname{Re} \operatorname{tr}(A B C)$.

Proof. This follows from the previous proposition and the definition of the trace.

The reader will have noticed three trilinears in this section: the associator $[a, b, c]$, the real part $\operatorname{Re}((a b) c)$, and the real trace $\operatorname{Re} \operatorname{tr}(A B C)$. This is no coincidence, as they all relate to the star of the show, tri $\psi$. In fact:

$$
\operatorname{tri} \psi=\operatorname{Re} \operatorname{tr}\left(\psi^{\dagger}(\epsilon \cdot \psi) \psi\right)
$$

for some suitable matrices $\psi^{\dagger}, \epsilon \cdot \psi$ and $\psi$. Of course, we have not yet said how to construct these. We turn to this now.

\section{$3 \quad$ Vectors, Spinors and Intertwiners}

It is well-known [1, 12, 17, that given a normed division algebra $\mathbb{K}$ of dimension $n$, one can construct $(n+2)$-dimensional Minkowski spacetime as the space of $2 \times 2$ hermitian matrices with entries in $\mathbb{K}$, with the determinant giving the Minkowski metric. Spinors can then be described as elements of $\mathbb{K}^{2}$. Our goal here is to provide self-contained proofs of these facts, and then develop all the basic operations involving vectors, spinors and scalars using this language.

To begin, let $\mathbb{K}[m]$ denote the space of $m \times m$ matrices with entries in $\mathbb{K}$. Given $A \in \mathbb{K}[m]$, define its hermitian adjoint $A^{\dagger}$ to be its conjugate transpose:

$$
A^{\dagger}=\left(A^{*}\right)^{T} .
$$

We say such a matrix is hermitian if $A=A^{\dagger}$. Now take the $2 \times 2$ hermitian matrices:

$$
\mathfrak{h}_{2}(\mathbb{K})=\left\{\left(\begin{array}{cc}
t+x & y \\
y^{*} & t-x
\end{array}\right): t, x \in \mathbb{R}, y \in \mathbb{K}\right\} .
$$

This is an $(n+2)$-dimensional real vector space. Moreover, the usual formula for the determinant of a matrix gives the Minkowski norm on this vector space:

$$
-\operatorname{det}\left(\begin{array}{cc}
t+x & y \\
y^{*} & t-x
\end{array}\right)=-t^{2}+x^{2}+|y|^{2} .
$$


We insert a minus sign to obtain the signature $(n+1,1)$. Note this formula is unambiguous even if $\mathbb{K}$ is noncommutative or nonassociative.

It follows that $\operatorname{Spin}(n+1,1)$, the double cover of the Lorentz group $\mathrm{SO}_{0}(n+1,1)$, acts on $\mathfrak{h}_{2}(\mathbb{K})$ via determinant-preserving linear transformations. Since this is the 'vector' representation, we will often call $\mathfrak{h}_{2}(\mathbb{K})$ simply $V$. The Minkowski metric

$$
g: V \otimes V \rightarrow \mathbb{R}
$$

is given by

$$
g(A, A)=-\operatorname{det}(A)
$$

There is also a nice formula for the inner product of two different vectors. This involves the trace reversal of $A \in \mathfrak{h}_{2}(\mathbb{K})$, introduced by Schray [14] and defined as follows:

$$
\tilde{A}=A-(\operatorname{tr} A) 1 \text {. }
$$

Note we indeed have $\operatorname{tr}(\tilde{A})=-\operatorname{tr}(A)$. Also note that

$$
A=\left(\begin{array}{cc}
t+x & y \\
y^{*} & t-x
\end{array}\right) \quad \Longrightarrow \quad \tilde{A}=\left(\begin{array}{cc}
-t+x & y \\
y^{*} & -t-x
\end{array}\right)
$$

so trace reversal is really time reversal. Moreover:

Proposition 5. For any vectors $A, B \in V=\mathfrak{h}_{2}(K)$, we have

$$
A \tilde{A}=\tilde{A} A=-\operatorname{det}(A) 1
$$

and

$$
\frac{1}{2} \operatorname{Re} \operatorname{tr}(A \tilde{B})=\frac{1}{2} \operatorname{Re} \operatorname{tr}(\tilde{A} B)=g(A, B)
$$

Proof. We check the first equation by a quick calculation. Taking the real trace and dividing by 2 gives

$$
\frac{1}{2} \operatorname{Re} \operatorname{tr}(A \tilde{A})=\frac{1}{2} \operatorname{Re} \operatorname{tr}(\tilde{A} A)=-\operatorname{det}(A)=g(A, A) .
$$

Then we use the polarization identity, which says that two symmetric bilinear forms that give the same quadratic form must be equal.

Next we consider spinors. As real vector spaces, the spinor representations $S_{+}$and $S_{-}$are both just $\mathbb{K}^{2}$. However, they differ as representations of $\operatorname{Spin}(n+1,1)$. To construct these representations, we begin by defining ways for vectors to act on spinors:

$$
\begin{aligned}
& \gamma: \quad V \otimes S_{+} \quad \rightarrow \quad S_{-} \\
& A \otimes \psi \quad \mapsto A \psi .
\end{aligned}
$$

and

$$
\begin{aligned}
\tilde{\gamma}: \quad V \otimes S_{-} & \rightarrow S_{+} \\
A \otimes \psi & \mapsto \tilde{A} \psi .
\end{aligned}
$$

We can also think of these as maps that send elements of $V$ to linear operators:

$$
\begin{array}{ll}
\gamma: & V \rightarrow \operatorname{Hom}\left(S_{+}, S_{-}\right), \\
\tilde{\gamma}: & V \rightarrow \operatorname{Hom}\left(S_{-}, S_{+}\right) .
\end{array}
$$

Here a word of caution is needed: since $\mathbb{K}$ may be nonassociative, $2 \times 2$ matrices with entries in $\mathbb{K}$ cannot be identified with linear operators on $\mathbb{K}^{2}$ in the usual way. They certainly induce linear operators via left multiplication:

$$
L_{A}(\psi)=A \psi .
$$


Indeed, this is how $\gamma$ and $\tilde{\gamma}$ turn elements of $V$ into linear operators:

$$
\begin{aligned}
& \gamma(A)=L_{A}, \\
& \tilde{\gamma}(A)=L_{\tilde{A}} .
\end{aligned}
$$

However, because of nonassociativity, composing such linear operators is different from multiplying the matrices:

$$
L_{A} L_{B}(\psi)=A(B \psi) \neq(A B) \psi=L_{A B}(\psi) .
$$

Since vectors act on elements of $S_{+}$to give elements of $S_{-}$and vice versa, they map the space $S_{+} \oplus S_{-}$to itself. This gives rise to an action of the Clifford algebra $\operatorname{Cliff}(V)$ on $S_{+} \oplus S_{-}$:

Proposition 6. The vectors $V=\mathfrak{h}_{2}(\mathbb{K})$ act on the spinors $S_{+} \oplus S_{-}=\mathbb{K}^{2} \oplus \mathbb{K}^{2}$ via the map

$$
\Gamma: V \rightarrow \operatorname{End}\left(S_{+} \oplus S_{-}\right)
$$

given by

$$
\Gamma(A)(\psi, \phi)=(\widetilde{A} \phi, A \psi)
$$

Furthermore, $\Gamma(A)$ satisfies the Clifford algebra relation:

$$
\Gamma(A)^{2}=g(A, A) 1
$$

and so extends to a homomorphism $\Gamma: \operatorname{Cliff}(V) \rightarrow \operatorname{End}\left(S_{+} \oplus S_{-}\right)$, i.e. a representation of the Clifford algebra $\operatorname{Cliff}(V)$ on $S_{+} \oplus S_{-}$.

Proof. Suppose $A \in V$ and $\Psi=(\psi, \phi) \in S_{+} \oplus S_{-}$. We need to check that

$$
\Gamma(A)^{2}(\Psi)=-\operatorname{det}(A) \Psi .
$$

Here we must be mindful of nonassociativity: we have

$$
\Gamma(A)^{2}(\Psi)=(\tilde{A}(A \psi), A(\tilde{A} \phi)) .
$$

Yet it is easy to check that the expressions $\tilde{A}(A \psi)$ and $A(\tilde{A} \phi)$ involve multiplying at most two different nonreal elements of $\mathbb{K}$. These associate, since $\mathbb{K}$ is alternative, so in fact

$$
\Gamma(A)^{2}(\Psi)=((\tilde{A} A) \psi,(A \tilde{A}) \phi) .
$$

To conclude, we use Proposition 5 ,

The action of a vector swaps $S_{+}$and $S_{-}$, so acting by vectors twice sends $S_{+}$to itself and $S_{-}$to itself. This means that while $S_{+}$and $S_{-}$are not modules for the Clifford algebra Cliff( $\left.V\right)$, they are both modules for the even part of the Clifford algebra, generated by products of pairs of vectors. The group $\operatorname{Spin}(n+1,1)$ lives in this even part. Indeed, call a vector $A$ such that $g(A, A)= \pm 1 \mathrm{a}$ unit vector. It is well known that the group in $\mathrm{Cliff}_{0}(V)$ generated by products of pairs of unit vectors is a double cover of $\mathrm{SO}(n+1,1)$, and thus its identity component is the double cover of $\mathrm{SO}_{0}(n+1,1)$. This identity component is therefore $\operatorname{Spin}(n+1,1)$.

While we will not need this in what follows, one can check that:

- When $\mathbb{K}=\mathbb{R}, S_{+} \cong S_{-}$is the Majorana spinor representation of $\operatorname{Spin}(2,1)$.

- When $\mathbb{K}=\mathbb{C}, S_{+} \cong S_{-}$is the Majorana spinor representation of $\operatorname{Spin}(3,1)$.

- When $\mathbb{K}=\mathbb{H}, S_{+}$and $S_{-}$are the Weyl spinor representations of $\operatorname{Spin}(5,1)$.

- When $\mathbb{K}=\mathbb{O}, S_{+}$and $S_{-}$are the Majorana-Weyl spinor representations of $\operatorname{Spin}(9,1)$. 
This counts as a consistency check, because these are precisely the kinds of spinor representations that go into pure super-Yang-Mills theory. But it is important to note that the differences between these spinor representations are irrelevant to our argument. What matters is how they are the same-they can all be defined on $\mathbb{K}^{2}$.

Now that we have representations of $\operatorname{Spin}(n+1,1)$ on $V, S_{+}$and $S_{-}$, we need to develop the $\operatorname{Spin}(n+1,1)$-equivariant maps that relate them. Ultimately, to define the Lagrangian for pure super-Yang-Mills theory, we need:

- An invariant pairing:

$$
\langle-,-\rangle: S_{+} \otimes S_{-} \rightarrow \mathbb{R} .
$$

- An equivariant map that turns pairs of spinors into vectors:

$$
\cdot: S_{ \pm} \otimes S_{ \pm} \rightarrow V
$$

Another name for an equivariant map between group representations is an 'intertwining operator'. As a first step, we show that the action of vectors on spinors is itself an intertwining operator:

Proposition 7. The maps

$$
\begin{aligned}
\gamma: \quad V \otimes S_{+} & \rightarrow S_{-} \\
A \otimes \psi & \mapsto A \psi
\end{aligned}
$$

and

$$
\begin{aligned}
\tilde{\gamma}: \quad V \otimes S_{-} & \rightarrow S_{+} \\
A \otimes \psi & \mapsto \tilde{A} \psi
\end{aligned}
$$

are equivariant with respect to the action of $\operatorname{Spin}(n+1,1)$.

Proof. Both $\gamma$ and $\tilde{\gamma}$ are restrictions of the map

$$
\Gamma: V \otimes\left(S_{+} \oplus S_{-}\right) \rightarrow S_{+} \oplus S_{-},
$$

so it suffices to check that $\Gamma$ is equivariant. Indeed, an element $g \in \operatorname{Spin}(n+1,1)$ acts on $V$ by conjugation on $V \subseteq \operatorname{Cliff}(V)$, and it acts on $S_{+} \oplus S_{-}$by $\Gamma(g)$. Thus, we compute:

$$
\Gamma\left(g A g^{-1}\right) \Gamma(g) \Psi=\Gamma(g)(\Gamma(A) \Psi),
$$

for any $\Psi \in S_{+} \oplus S_{-}$. Here it is important to note that the conjugation $g \mathrm{Ag}^{-1}$ is taking place in the associative algebra $\operatorname{Cliff}(V)$, not in the algebra of matrices. This equation says that $\Gamma$ is indeed $\operatorname{Spin}(n+1,1)$-equivariant, as claimed.

Now we exhibit the key tool: the pairing between $S_{+}$and $S_{-}$:

Proposition 8. The pairing

$$
\begin{aligned}
\langle-,-\rangle: \quad S_{+} \otimes S_{-} & \rightarrow \mathbb{R} \\
\psi \otimes \phi & \mapsto \operatorname{Re}\left(\psi^{\dagger} \phi\right)
\end{aligned}
$$

is invariant under the action of $\operatorname{Spin}(n+1,1)$.

Proof. Given $A \in V$, we use the fact that the associator is purely imaginary to show that

$$
\operatorname{Re}\left((\tilde{A} \phi)^{\dagger}(A \psi)\right)=\operatorname{Re}\left(\left(\phi^{\dagger} \tilde{A}\right)(A \psi)\right)=\operatorname{Re}\left(\phi^{\dagger}(\tilde{A}(A \psi))\right) .
$$

As in the proof of the Clifford relation, it is easy to check that the column vector $\tilde{A}(A \psi)$ involves at most two nonreal elements of $\mathbb{K}$ and equals $g(A, A) \psi$. So:

$$
\langle\tilde{\gamma}(A) \phi, \gamma(A) \psi\rangle=g(A, A)\langle\psi, \phi\rangle .
$$


In particular when $A$ is a unit vector, acting by $A$ swaps the order of $\psi$ and $\phi$ and changes the sign at most. In fact, this implies our result, though we need a more explicit presentation of $\operatorname{Spin}(n+1,1)$ to see this. Proposition 5.4.8 of Varadarajan [18 tells us that $\operatorname{Spin}(n+1,1)$ is the group generated by products of even numbers of unit vectors, an even number of which satisfy $g(A, A)=-1$ :

$$
\operatorname{Spin}(n+1,1)=\left\langle A_{1} \cdots A_{2 p} B_{1} \cdots B_{2 q}: A_{i}, B_{j} \in V, g\left(A_{i}, A_{i}\right)=1, g\left(B_{j}, B_{j}\right)=-1\right\rangle .
$$

By the computation above, this implies that $\langle-,-\rangle$ is invariant under $\operatorname{Spin}(n+1,1)$.

With this pairing in hand, there is a manifestly equivariant way to turn a pair of spinors into a vector. Given $\psi, \phi \in S_{+}$, there is a unique vector $\psi \cdot \phi$ whose inner product with any vector $A$ is given by

$$
g(\psi \cdot \phi, A)=\langle\psi, \gamma(A) \phi\rangle .
$$

Similarly, given $\psi, \phi \in S_{-}$, we define $\psi \cdot \phi \in V$ by demanding

$$
g(\psi \cdot \phi, A)=\langle\tilde{\gamma}(A) \psi, \phi\rangle
$$

for all $A \in V$. This gives us maps

$$
S_{ \pm} \otimes S_{ \pm} \rightarrow V
$$

which are manifestly equivariant.

On the other hand, because $S_{ \pm}=\mathbb{K}^{2}$ and $V=\mathfrak{h}_{2}(\mathbb{K})$, there is also a naive way to turn a pair of spinors into a vector using matrix operations: just multiply the column vector $\psi$ by the row vector $\phi^{\dagger}$ and then take the hermitian part:

$$
\psi \phi^{\dagger}+\phi \psi^{\dagger} \in \mathfrak{h}_{2}(\mathbb{K})
$$

or perhaps its trace reversal:

$$
\widetilde{\psi \phi^{\dagger}+\phi} \psi^{\dagger} \in \mathfrak{h}_{2}(\mathbb{K})
$$

In fact, these naive guesses match the manifestly equivariant approach described above:

Proposition 9. The maps $\cdot: S_{ \pm} \otimes S_{ \pm} \rightarrow V$ are given by:

$$
\begin{array}{rll}
: S_{+} \otimes S_{+} & \rightarrow V \\
\psi \otimes \phi & \mapsto \psi \phi^{\dagger}+\phi \psi^{\dagger} \\
\therefore: S_{-} \otimes S_{-} & \rightarrow V \\
\psi \otimes \phi & \mapsto \psi \phi^{\dagger}+\phi \psi^{\dagger} .
\end{array}
$$

These maps are equivariant with respect to the action of $\operatorname{Spin}(n+1,1)$.

Proof. First suppose $\psi, \phi \in S_{+}$. We have already seen that the map $\cdot: S_{+} \otimes S_{+} \rightarrow V$ is equivariant. We only need to show that this map has the desired form. We start by using some definitions:

$$
g(\psi \cdot \phi, A)=\langle\psi, \gamma(A) \phi\rangle=\operatorname{Re}\left(\psi^{\dagger}(A \phi)\right)=\operatorname{Re} \operatorname{tr}\left(\psi^{\dagger} A \phi\right) .
$$

We thus have

$$
g(\psi \cdot \phi, A)=\operatorname{Re} \operatorname{tr}\left(\psi^{\dagger} A \phi\right)=\operatorname{Re} \operatorname{tr}\left(\phi^{\dagger} A \psi\right),
$$

where in the last step we took the adjoint of the inside. Applying the cyclic property of the real trace, we obtain

$$
g(\psi \cdot \phi, A)=\operatorname{Re} \operatorname{tr}\left(\phi \psi^{\dagger} A\right)=\operatorname{Re} \operatorname{tr}\left(\psi \phi^{\dagger} A\right) .
$$

Averaging gives

$$
g(\psi \cdot \phi, A)=\frac{1}{2} \operatorname{Re} \operatorname{tr}\left(\left(\psi \phi^{\dagger}+\phi \psi^{\dagger}\right) A\right) .
$$


On the other hand, Proposition 5 implies that

$$
\left.g(\psi \cdot \phi, A)=\frac{1}{2} \operatorname{Re} \operatorname{tr}(\widetilde{(\psi \cdot \phi}) A\right) .
$$

Since both these equations hold for all $A$, we must have

$$
\widetilde{\psi \cdot \phi}=\psi \phi^{\dagger}+\phi \psi^{\dagger}
$$

Doing trace reversal twice gets us back where we started, so

$$
\psi \cdot \phi=\psi \widehat{\phi^{\dagger}+\phi} \psi^{\dagger}
$$

as desired. A similar calculation shows that if $\psi, \phi \in S_{-}$, then $\psi \cdot \phi=\psi \phi^{\dagger}+\phi \psi^{\dagger}$.

\begin{tabular}{ccc}
\hline \multicolumn{1}{l}{ Map } & Division algebra notation & Index notation \\
\hline$g: V \otimes V \rightarrow \mathbb{R}$ & $\frac{1}{2} \operatorname{Re} \operatorname{tr}(A \tilde{B})$ & $A^{\mu} B_{\mu}$ \\
$\gamma: V \otimes S_{+} \rightarrow S_{-}$ & $A \psi$ & $\gamma_{\mu} A^{\mu} \psi$ \\
$\tilde{\gamma}: V \otimes S_{-} \rightarrow S_{+}$ & $\tilde{A} \psi$ & $\tilde{\gamma} \mu A^{\mu} \psi$ \\
$\cdot: S_{+} \otimes S_{+} \rightarrow V$ & $\psi \phi^{\dagger}+\phi \psi^{\dagger}$ & $\bar{\psi} \gamma^{\mu} \phi$ \\
$\cdot: S_{-} \otimes S_{-} \rightarrow V$ & $\psi \phi^{\dagger}+\phi \psi^{\dagger}$ & $\bar{\psi} \tilde{\gamma}^{\mu} \phi$ \\
$\langle-,-\rangle: S_{+} \otimes S_{-} \rightarrow \mathbb{R}$ & $\operatorname{Re}\left(\psi^{\dagger} \phi\right)$ & $\bar{\psi} \phi$ \\
\hline
\end{tabular}

Table 1: Division algebra notation vs. index notation

We can summarize our work so far with a table of the basic bilinear maps involving vectors, spinors and scalars. Table 1 shows how to translate between division algebra notation and something more closely resembling standard physics notation. In this table the adjoint spinor $\bar{\psi}$ denotes the spinor dual to $\psi$ under the pairing $\langle-,-\rangle$. The gamma matrix $\gamma^{\mu}$ denotes a Clifford algebra generator acting on $S_{+}$, while $\tilde{\gamma}^{\mu}$ denotes the same element acting on $S_{-}$. Of course $\tilde{\gamma}$ is not standard physics notation; the standard notation for this depends on which of the four cases we are considering: $\mathbb{R}$, $\mathbb{C}, \mathbb{H}$ or $\mathbb{O}$.

\section{The 3- $\psi$ 's Rule}

Now we prove the fundamental identity that makes supersymmetry tick in dimensions $3,4,6$, and 10. This identity was dubbed the ' $3-\psi$ 's rule' by Schray [14. The following proof is based on an argument in the appendix of the paper by Dray, Janesky and Manogue [5]. Note that it is really the alternative law, rather than the normed division algebra axioms, that does the job:

Theorem 10. Suppose $\psi \in S_{+}$. Then $(\psi \cdot \psi) \psi=0$. Similarly, if $\phi \in S_{-}$, then $(\widetilde{\phi} \cdot \phi) \phi=0$.

Proof. Suppose $\psi \in S_{+}$. By definition,

$$
(\psi \cdot \psi) \psi=2\left(\widetilde{\psi \psi^{\dagger}}\right) \psi=2\left(\psi \psi^{\dagger}-\operatorname{tr}\left(\psi \psi^{\dagger}\right) 1\right) \psi .
$$

It is easy to check that $\operatorname{tr}\left(\psi \psi^{\dagger}\right)=\psi^{\dagger} \psi$, so

$$
(\psi \cdot \psi) \psi=2\left(\left(\psi \psi^{\dagger}\right) \psi-\left(\psi^{\dagger} \psi\right) \psi\right)
$$


Since $\psi^{\dagger} \psi$ is a real number, it commutes with $\psi$ :

$$
(\psi \cdot \psi) \psi=2\left(\left(\psi \psi^{\dagger}\right) \psi-\psi\left(\psi^{\dagger} \psi\right)\right) .
$$

Since $\mathbb{K}$ is alternative, every subalgebra of $\mathbb{K}$ generated by two elements is associative. Since $\psi \in \mathbb{K}^{2}$ is built from just two elements of $\mathbb{K}$, the right-hand side vanishes. The proof of the identity for $\phi \in S_{-}$is similar.

It will be useful to state this result in a somewhat more elaborate form. To save space we only give this version for spinors in $S_{+}$, though an analogous result holds for spinors in $S_{-}$:

Theorem 11. Define a map

$$
\begin{aligned}
T: \quad S_{+} \otimes S_{+} \otimes S_{+} & \rightarrow S_{-} \\
\psi \otimes \phi \otimes \chi & \mapsto(\psi \cdot \phi) \chi+(\phi \cdot \chi) \psi+(\chi \cdot \psi) \phi .
\end{aligned}
$$

Then $T=0$.

Proof. It is easy to check that $\psi \cdot \phi=\phi \cdot \psi$ for all $\psi, \phi \in S_{+}$, so the map $T$ is completely symmetric in its three arguments. Just as any symmetric bilinear form $B(x, y)$ can be recovered from the corresponding quadratic form $B(x, x)$ by polarization, so too can any symmetric trilinear form be recovered from the corresponding cubic form. Since $T(\psi, \psi, \psi)=0$ by Theorem 10, it follows that $T=0$.

To see how this theorem is the key to supersymmetry for super-Yang-Mills theory, we need a little superalgebra.

\section{Superalgebra}

So far we have used normed division algebras to construct a number of algebraic structures: vectors as elements of $\mathfrak{h}_{2}(\mathbb{K})$, spinors as elements of $\mathbb{K}^{2}$, and the various bilinear maps involving vectors, spinors, and scalars. However, to describe supersymmetry, we also need superalgebra. Specifically, we need anticommuting spinors. Physically, this is because spinors are fermions, so we need them to satisfy anticommutation relations. Mathematically, this means that we will do our algebra in the category of 'super vector spaces', SuperVect, rather than the category of vector spaces, Vect.

A super vector space is a $\mathbb{Z}_{2}$-graded vector space $V=V_{0} \oplus V_{1}$ where $V_{0}$ is called the even or bosonic part, and $V_{1}$ is called the odd or fermionic part. Like Vect, SuperVect is a symmetric monoidal category [2]. It has:

- $\mathbb{Z}_{2}$-graded vector spaces as objects;

- Grade-preserving linear maps as morphisms;

- A tensor product $\otimes$ that has the following grading: if $V=V_{0} \oplus V_{1}$ and $W=W_{0} \oplus W_{1}$, then $(V \otimes W)_{0}=\left(V_{0} \otimes W_{0}\right) \oplus\left(V_{1} \otimes W_{1}\right)$ and $(V \otimes W)_{1}=\left(V_{0} \otimes W_{1}\right) \oplus\left(V_{1} \otimes W_{0}\right) ;$

- A braiding

$$
B_{V, W}: V \otimes W \rightarrow W \otimes V
$$

defined as follows: $v \in V$ and $w \in W$ are of grade $p$ and $q$, then

$$
B_{V, W}(v \otimes w)=(-1)^{p q} w \otimes v .
$$


The braiding encodes the 'the rule of signs': in any calculation, when two odd elements are interchanged, we introduce a minus sign.

In what follows we treat the normed division algebra $\mathbb{K}$ as an odd super vector space. This turns out to force the spinor representations $S_{ \pm}$to be odd and the vector representation $V$ to be even, as follows.

There is an obvious notion of direct sums for super vector spaces, with

$$
(V \oplus W)_{0}=V_{0} \oplus W_{0}, \quad(V \oplus W)_{1}=V_{1} \oplus W_{1}
$$

and also an obvious notion of duals, with

$$
\left(V^{*}\right)_{0}=\left(V_{0}\right)^{*}, \quad\left(V^{*}\right)_{1}=\left(V_{1}\right)^{*} .
$$

We say a super vector space $V$ is even if it equals its even part $\left(V=V_{0}\right)$, and odd if it equals its odd part $\left(V=V_{1}\right)$. Any subspace $U \subseteq V$ of an even (resp. odd) super vector space becomes a super vector space which is again even (resp. odd).

We treat the spinor representations $S_{ \pm}$as super vector spaces using the fact that they are the direct sum of two copies of $\mathbb{K}$. Since $\mathbb{K}$ is odd, so are $S_{+}$and $S_{-}$. Since $\mathbb{K}^{2}$ is odd, so is its dual. This in turn forces the space of linear maps from $\mathbb{K}^{2}$ to itself, $\operatorname{End}\left(\mathbb{K}^{2}\right)=\mathbb{K}^{2} \otimes\left(\mathbb{K}^{2}\right)^{*}$, to be even. This even space contains the $2 \times 2$ matrices $\mathbb{K}[2]$ as the subspace of maps realized by left multiplication:

$$
\begin{aligned}
\mathbb{K}[2] & \hookrightarrow \operatorname{End}\left(\mathbb{K}^{2}\right) \\
A & \mapsto L_{A} .
\end{aligned}
$$

$\mathbb{K}[2]$ is thus even. Finally, this forces the subspace of hermitian $2 \times 2$ matrices, $\mathfrak{h}_{2}(\mathbb{K})$, to be even. So, the vector representation $V$ is even. All this matches the usual rules in physics, where spinors are fermionic and vectors are bosonic.

\section{Super-Yang-Mills Theory}

We are now ready to give a division algebra interpretation of the pure super-Yang-Mills Lagrangian

$$
L=-\frac{1}{4}\langle F, F\rangle+\frac{1}{2}\left\langle\psi, \not D_{A} \psi\right\rangle
$$

and use this to prove its supersymmetry. For simplicity, we shall work over Minkowski spacetime, $M$. This allows us to treat all bundles as trivial, sections as functions, and connections as $\mathfrak{g}$-valued 1 -forms.

At the outset, we fix an invariant inner product on $\mathfrak{g}$, the Lie algebra of a semisimple Lie group $G$. We shall use the following standard tools from differential geometry to construct $L$, none of which need involve spinors or division algebra technology:

- A connection $A$ on a principal $G$-bundle over $M$. Since the bundle is trivial we think of this connection as a $\mathfrak{g}$-valued 1-form.

- The exterior covariant derivative $d_{A}=d+[A,-]$ on $\mathfrak{g}$-valued $p$-forms.

- The curvature $F=d A+\frac{1}{2}[A, A]$, which is a $\mathfrak{g}$-valued 2 -form.

- The usual pointwise inner product $\langle F, F\rangle$ on $\mathfrak{g}$-valued 2-forms, defined using the Minkowski metric on $M$ and the invariant inner product on $\mathfrak{g}$.

We also need the following spinorial tools. Recall from the preceding section that $S_{+}$and $S_{-}$are odd objects in SuperVect. So, whenever we switch two spinors, we introduce a minus sign. 
- A $\mathfrak{g}$-valued section $\psi$ of a spin bundle over $M$. Note that this is, in fact, just a function:

$$
\psi: M \rightarrow S_{ \pm} \otimes \mathfrak{g} .
$$

We call the collection of all such functions $\Gamma\left(S_{ \pm} \otimes \mathfrak{g}\right)$.

- The covariant Dirac operator $\not D_{A}$ derived from the connection $A$. Of course,

$$
\not D_{A}: \Gamma\left(S_{ \pm} \otimes \mathfrak{g}\right) \rightarrow \Gamma\left(S_{\mp} \otimes \mathfrak{g}\right)
$$

and in fact,

$$
\not D_{A}=\not \partial+A
$$

- A bilinear pairing

$$
\langle-,-\rangle: \Gamma\left(S_{+} \otimes \mathfrak{g}\right) \otimes \Gamma\left(S_{-} \otimes \mathfrak{g}\right) \rightarrow C^{\infty}(M)
$$

built pointwise using our pairing

$$
\langle-,-\rangle: S_{+} \otimes S_{-} \rightarrow \mathbb{R}
$$

and the invariant inner product on $\mathfrak{g}$.

The basic fields in our theory are a connection on a principal $G$-bundle, which we think of as a $\mathfrak{g}$-valued 1-form:

$$
A: M \rightarrow V^{*} \otimes \mathfrak{g} .
$$

and a $\mathfrak{g}$-valued spinor field, which we think of as a $S_{+} \otimes \mathfrak{g}$-valued function on $M$ :

$$
\psi: M \rightarrow S_{+} \otimes \mathfrak{g} .
$$

All our arguments would work just as well with $S_{-}$replacing $S_{+}$.

To show that $L$ is supersymmetric, we need to show $\delta L$ is a total divergence when $\delta$ is the following supersymmetry transformation:

$$
\begin{aligned}
\delta A & =\epsilon \cdot \psi \\
\delta \psi & =\frac{1}{2} F \epsilon
\end{aligned}
$$

where $\epsilon$ is an arbitrary constant spinor field, treated as odd, but not $\mathfrak{g}$-valued. By a supersymmetry transformation we mean that computationally we treat $\delta$ as a derivation. So, it is linear:

$$
\delta(\alpha f+\beta g)=\alpha \delta f+\beta \delta g
$$

where $\alpha, \beta \in \mathbb{R}$, and it satisfies the product rule:

$$
\delta(f g)=\delta(f) g+f \delta g .
$$

For a more formal definition of 'supersymmetry transformation' see [6].

The above equations require further explanation. The dot in $\epsilon \cdot \psi$ denotes an operation that combines the spinor $\epsilon$ with the $\mathfrak{g}$-valued spinor $\psi$ to produce a $\mathfrak{g}$-valued 1-form. We build this from our basic intertwiner

$$
\cdot S_{+} \otimes S_{+} \rightarrow V
$$

We identify $V$ with $V^{*}$ using the Minkowski inner product $g$, obtaining

$$
\cdot: S_{+} \otimes S_{+} \rightarrow V^{*} .
$$


Then we tensor both sides with $\mathfrak{g}$. This gives us a way to act by a spinor field on a $\mathfrak{g}$-valued spinor field to obtain a $\mathfrak{g}$-valued 1-form. We take the liberty of also denoting this with a dot:

$$
\cdot: \Gamma\left(S_{+}\right) \otimes \Gamma\left(S_{+} \otimes \mathfrak{g}\right) \rightarrow \Omega^{1}(M, \mathfrak{g}) .
$$

We also need to explain how the 2-form $F$ acts on the constant spinor field $\epsilon$. Using the Minkowski metric, we can identify differential forms on $M$ with sections of the Clifford algebra bundle over $M$ :

$$
\Omega^{*}(M) \cong \operatorname{Cliff}(M) .
$$

Using this, differential forms act on spinor fields. Tensoring with $\mathfrak{g}$, we obtain a way for $\mathfrak{g}$-valued differential forms like $F$ to act on spinor fields like $\epsilon$ to give $\mathfrak{g}$-valued spinor fields like $F \epsilon$.

Let us now apply the supersymmetry transformation to each term in the Lagrangian. First, the bosonic term:

Proposition 12. The bosonic term has:

$$
\delta\langle F, F\rangle=2(-1)^{n+1}\left\langle\psi,\left(\star d_{A} \star F\right) \epsilon\right\rangle+\text { divergence. }
$$

Proof. By the symmetry of the inner product, we get:

$$
\delta\langle F, F\rangle=2\langle F, \delta F\rangle .
$$

Using the handy formula $\delta F=d_{A} \delta A$, we have:

$$
\langle F, \delta F\rangle=\left\langle F, d_{A} \delta A\right\rangle .
$$

Now the adjoint of the operator $d_{A}$ is $\star d_{A} \star$, up to a pesky sign: if $\nu$ is a $\mathfrak{g}$-valued $(p-1)$-form and $\mu$ is a $\mathfrak{g}$-valued $p$-form, we have

$$
\left\langle\mu, d_{A} \nu\right\rangle=(-1)^{d p+d+1+s}\left\langle\star d_{A} \star \mu, \nu\right\rangle+\text { divergence }
$$

where $d$ is the dimension of spacetime and $s$ is the signature, i.e., the number of minus signs in the diagonalized metric. It follows that

$$
\langle F, \delta F\rangle=\left\langle F, d_{A} \delta A\right\rangle=(-1)^{n}\left\langle\star d_{A} \star F, \delta A\right\rangle+\text { divergence }
$$

where $n$ is the dimension of $\mathbb{K}$. By the definition of $\delta A$, we get

$$
\left\langle\star d_{A} \star F, \delta A\right\rangle=\left\langle\star d_{A} \star F, \epsilon \cdot \psi\right\rangle .
$$

Now we can use division algebra technology to show:

$$
\left\langle\star d_{A} \star F, \epsilon \cdot \psi\right\rangle=\frac{1}{2} \operatorname{Re} \operatorname{tr}\left(\left(\star d_{A} \star F\right)\left(\epsilon \psi^{\dagger}+\psi \epsilon^{\dagger}\right)\right)=-\left\langle\psi,\left(\star d_{A} \star F\right) \epsilon\right\rangle,
$$

using the cyclic property of the real trace in the last step, and introducing a minus sign in accordance with the sign rule. Putting everything together, we obtain the desired result.

Even though this proposition involved the bosonic term only, division algebra technology was still a useful tool in its proof. This is even more true in the next proposition, which deals with the the fermionic term:

Proposition 13. The fermionic term has:

$$
\delta\left\langle\psi, \not D_{A} \psi\right\rangle=\left\langle\psi, \not D_{A}(F \epsilon)\right\rangle+\operatorname{tri} \psi+\text { divergence }
$$

where

$$
\operatorname{tri} \psi=\langle\psi,(\epsilon \cdot \psi) \psi\rangle
$$


Proof. It is easy to compute:

$$
\delta\left\langle\psi, \not D_{A} \psi\right\rangle=\left\langle\delta \psi, \not D_{A} \psi\right\rangle+\left\langle\psi, \delta \not D_{A} \psi\right\rangle+\left\langle\psi, \not D_{A} \delta \psi\right\rangle .
$$

Now we insert $\delta \not D_{A}=\delta A=\epsilon \cdot \psi$, and thus see that the penultimate term is the trilinear one:

$$
\operatorname{tri} \psi=\langle\psi,(\epsilon \cdot \psi) \psi\rangle \text {. }
$$

So, let us concern ourselves with the remaining terms:

$$
\left\langle\delta \psi, \not D_{A} \psi\right\rangle+\left\langle\psi, \not D_{A} \delta \psi\right\rangle \text {. }
$$

A computation using the product rule shows that the divergence of the 1 -form $\psi \cdot \phi$ is given by $-\left\langle\phi, \not D_{A} \psi\right\rangle+\left\langle\psi, \not D_{A} \phi\right\rangle$, where the minus sign on the first term arises from using the sign rule with these odd spinors. In the terms under consideration, we can use this identity to move $\not_{A}$ onto $\delta \psi$ :

$$
\left\langle\delta \psi, \not D_{A} \psi\right\rangle+\left\langle\psi, \not D_{A} \delta \psi\right\rangle=2\left\langle\psi, \not D_{A} \delta \psi\right\rangle+\text { divergence. }
$$

Substituting $\delta \psi=\frac{1}{2} F \epsilon$, we obtain the desired result.

Using these two propositions, it is immediate that

$$
\begin{aligned}
\delta L & =-\frac{1}{4} \delta\langle F, F\rangle+\frac{1}{2} \delta\left\langle\psi, \not D_{A} \psi\right\rangle \\
& =\frac{1}{2}(-1)^{n}\left\langle\psi,\left(\star d_{A} \star F\right) \epsilon\right\rangle+\frac{1}{2}\left\langle\psi, \not D_{A}(F \epsilon)\right\rangle+\frac{1}{2} \operatorname{tri} \psi+\text { divergence }
\end{aligned}
$$

All that remains to show is that $\not D_{A}(F \epsilon)=(-1)^{n+1}\left(\star d_{A} \star F\right) \epsilon$. Indeed, Snygg shows (Eq. 7.6 in [16]) that for an ordinary, non-g-valued $p$-form $F$

$$
\not \partial(F \epsilon)=(d F) \epsilon+(-1)^{d+d p+s}(\star d \star F) \epsilon
$$

where $d$ is the dimension of spacetime and $s$ is the signature. This is easily generalized to covariant derivatives and $\mathfrak{g}$-valued $p$-forms:

$$
\not D_{A}(F \epsilon)=\left(d_{A} F\right) \epsilon+(-1)^{d+d p+s}\left(\star d_{A} \star F\right) \epsilon .
$$

In particular, when $F$ is the curvature 2 -form, the first term vanishes by the Bianchi identity $d_{A} F=$ 0 , and we are left with:

$$
\not D_{A}(F \epsilon)=(-1)^{n+1}\left(\star d_{A} \star F\right) \epsilon
$$

where $n$ is the dimension of $\mathbb{K}$. We have thus shown:

Proposition 14. Under supersymmetry transformations, the Lagrangian L has:

$$
\delta L=\frac{1}{2} \operatorname{tri} \psi+\text { divergence. }
$$

The above result actually holds in every dimension, though our proof used division algebras and was thus adapted to the dimensions of interest: $3,4,6$, and 10 . The next result is where division algebra technology becomes really crucial:

Proposition 15. For Minkowski spacetimes of dimensions 3, 4, 6, and 10, tri $\psi=0$. 
Proof. At each point, we can write

$$
\psi=\sum \psi^{a} \otimes g_{a},
$$

where $\psi^{a} \in S_{+}$and $g_{a} \in \mathfrak{g}$. When we insert this into tri $\psi$, we see that

$$
\operatorname{tri} \psi=\sum\left\langle\psi^{a},\left(\epsilon \cdot \psi^{b}\right) \psi^{c}\right\rangle\left\langle g_{a},\left[g_{b}, g_{c}\right]\right\rangle .
$$

Since $\left\langle g_{a},\left[g_{b}, g_{c}\right]\right\rangle$ is totally antisymmetric, this implies tri $\psi=0$ for all $\epsilon$ if and only if the part of $\left\langle\psi^{a},\left(\epsilon \cdot \psi^{b}\right) \psi^{c}\right\rangle$ that is antisymmetric in $a, b$ and $c$ vanishes for all $\epsilon$. Yet these spinors are odd; for even spinors, we require the part of $\left\langle\psi^{a},\left(\epsilon \cdot \psi^{b}\right) \psi^{c}\right\rangle$ that is symmetric in $a, b$ and $c$ to vanish for all $\epsilon$.

Now let us bring in some division algebra technology to remove our dependence on $\epsilon$. While we do this, let us replace $\psi^{a}$ with $\psi, \psi^{b}$ with $\phi$, and $\psi^{c}$ with $\chi$ to lessen the clutter of indices. Substituting in the formulas from Table 1 we have

$$
\begin{aligned}
& \langle\psi,(\epsilon \cdot \phi) \chi\rangle=\operatorname{Re}\left(\psi^{\dagger}\left(\widetilde{\phi^{\dagger}+\phi \epsilon^{\dagger}}\right) \chi\right) \\
& =\operatorname{Re} \operatorname{tr}\left(\psi^{\dagger}\left(\epsilon \phi^{\dagger}+\phi \epsilon^{\dagger}-\epsilon^{\dagger} \phi-\phi^{\dagger} \epsilon\right) \chi\right) \\
& =\langle\epsilon,(\psi \cdot \chi) \phi\rangle \text {, }
\end{aligned}
$$

where again we have employed the cyclic symmetry of the real trace, along with the identity:

$$
\operatorname{tr}\left(\epsilon \phi^{\dagger}+\phi \epsilon^{\dagger}\right)=\operatorname{Re} \operatorname{tr}\left(\epsilon \phi^{\dagger}+\phi \epsilon^{\dagger}\right)=\phi^{\dagger} \epsilon+\epsilon^{\dagger} \phi .
$$

This real quantity commutes and associates in any expression. So, if we seek to show that the part of $\langle\psi,(\epsilon \cdot \phi) \chi\rangle$ that is totally symmetric in $\psi, \phi$ and $\chi$ vanishes for all $\epsilon$, it is equivalent to show the totally symmetric part of $(\phi \cdot \chi) \psi$ vanishes. And since the dot operation in $\phi \cdot \chi$ is symmetric, this follows immediately from our main result, Theorem 11 .

\section{Acknowledgements}

We thank Geoffrey Dixon, Tevian Dray and Corinne Manogue for helpful conversations and correspondence. We also thank An Huang, Theo Johnson-Freyd, Greg Egan, and David Speyer for catching some errors. This work was partially supported by an FQXi grant.

\section{References}

[1] J. C. Baez, The octonions, Bull. Amer. Math. Soc. 39 (2002), 145-205. Also available as arXiv:math/0105155

[2] J. C. Baez and M. Stay, Physics, topology, logic and computation: a Rosetta Stone, to appear in New Structures For Physics, ed. Bob Coecke. Also available as arXiv:0903.0340.

[3] L. Brink, J. Schwarz and J. Scherk, Supersymmetric Yang-Mills theory, Nucl. Phys. B121 (1977), 77-92.

[4] K.-W. Chung and A. Sudbery, Octonions and the Lorentz and conformal groups of tendimensional space-time, Phys. Lett. B 198 (1987), 161-164.

[5] T. Dray, J. Janesky and C. A. Manogue, Octonionic hermitian matrices with non-real eigenvalues, Adv. Appl. Clifford Algebras 10 (2000), 193-216. Also available as arXiv:math/0006069.

[6] P. Deligne et al, eds., Quantum Fields and Strings: A Course for Mathematicians, Volume 1, Amer. Math. Soc., Providence, Rhode Island, 1999. 
[7] J. M. Evans, Supersymmetric Yang-Mills theories and division algebras, Nucl. Phys. B298 (1988), 92-108. Also available as 〈http://www-lib.kek.jp/cgi-bin/img_index?198801412).

[8] D. B. Fairlie and C. A. Manogue, A parameterization of the covariant superstring, Phys. Rev. D36 (1987), 475-479.

[9] M. Green and J. Schwarz, Covariant description of superstrings, Phys. Lett. B136 (1984), $367-370$.

[10] M. Green, J. Schwarz and E. Witten, Superstring Theory, Volume 1, Cambridge U. Press, Cambridge, 1987. Appendix 4.A: Super Yang-Mills theories, pp. 244-247. Section 5.1.2: The supersymmetric string action, pp. 253-255.

[11] A. Hurwitz, Über die Composition der quadratischen Formen von beliebig vielen Variabeln, Nachr. Ges. Wiss. Göttingen (1898), 309-316.

[12] T. Kugo and P. Townsend, Supersymmetry and the division algebras, Nucl. Phys. B221 (1983), 357-380. Also available at 〈http://www-lib.kek.jp/cgi-bin/img_index?198301032

[13] C. A. Manogue and A. Sudbery, General solutions of covariant superstring equations of motion, Phys. Rev. D 12 (1989), 4073-4077.

[14] J. Schray, The general classical solution of the superparticle, Class. Quant. Grav. 13 (1996), 27-38. Also available as arXiv:hep-th/9407045.

[15] R. D. Schafer, Introduction to Non-Associative Algebras, Dover, New York, 1995.

[16] J. Snygg, Clifford Algebra: a Computational Tool for Physicists, Oxford U. Press, Oxford, 1997.

[17] A. Sudbery, Division algebras, (pseudo)orthogonal groups and spinors, Jour. Phys. A17 (1984), 939-955.

[18] V. S. Varadarajan, Supersymmetry for Mathematicians, Amer. Math. Soc. Providence, 2004. Section 5.4: Spin groups and spin representations, pp. 192-203. 